Pacific Journal of Mathematics

REMARKS ON THE MAXIMUM PRINCIPLE FOR PARABOLIC 


\section{REMARKS ON THE MAXIMUM PRINCIPLE FOR PARABOLIC EQUATIONS AND ITS APPLICATIONS}

\section{AVNer Friedman}

Introduction. In [3] Nirenberg has proved maximum principles, both weak and strong, for parabolic equations. In $\S 1$ of this paper we give a generalization of his strong maximum principle (Theorem 1). Hopf [2] and Olainik [4] have proved that if $L u \geqq 0$ and $L$ is a linear elliptic operator of the second order, if the coefficient of $u$ in $L$ is nonpositive, and if $u$ ( $\equiv$ const.) assumes its positive maximum at a point $P^{\prime}$ (which necessarily belongs to the boundary) then $\partial u / \partial_{\nu}<0$, where $\nu$ is the inwardly directed normal. In $\S 2$ we extend this result to parabolic operators (Theorem 2). A further discussion of the assumptions made in Theorem 2 is given in $\S 3$. Application of Theorem 2 to the Neumann problem is given in $\S 4$. In $\S 5$ we apply the weak maximum principle to prove a uniqueness theorem for certain nonlinear parabolic equations with nonlinear boundary conditions, and thus extend the special case considered by Ficken [1]. An even more special case arises in the theory of diffusion (for references, see [1]).

\section{Consider the operator}

$$
L u \equiv \sum_{i, j=1}^{n} a_{i j}(x, t) \frac{\partial^{2} u}{\partial x_{i} \partial x_{j}}+\sum_{i=1}^{n} a_{i}(x, t) \frac{\partial u}{\partial x_{i}}+a(x, t) u-\frac{\partial u}{\partial t}
$$

with $a(x, t) \leqq 0$. Here, $(x, t)=\left(x_{1}, \cdots, x_{n}, t\right)$ varies in the closure $\bar{D}$ of a given $(n+1)$-dimensional domain $D$. Assume that $L$ is parabolic in $\bar{D}$, that is, for every real vector $\lambda \neq 0$ and for every $(x, t) \in \bar{D}$ we have

$$
\sum a_{i j}(x, t) \lambda_{i} \lambda_{j}>0 \text {. }
$$

All the coefficients of $L$ are assumed to be continuous in $\bar{D}$ and $u$ is assumed to be continuous in $\bar{D}$ and to have a continuous $t$-derivative and continuous second $x$-derivatives in $D$. From [3; Th. 5] it follows that, under the above assumptions, if $L u \geqq 0$ and if $u$ assumes its positive maximum at an interior point $P^{0}$, then $u \equiv$ const. in $S\left(P^{0}\right)$. Here, $S\left(P^{0}\right)$ denotes the set of all points $Q$ in $D$ which can be connected to $P^{0}$ by a simple continuous curve in $D$ along which the coordinate $t$ is non-decreasing from $Q$ to $P^{0}$. In the following theorem we consider the case

Received October 29, 1957. Prepared under ONR Contract Nonr-908 (09), NR 041037 with Indiana University. 
in which $P^{0}$ is a boundary point of $D$. We may assume that $P^{0}$ is the origin. Let $t=\varphi(x)$ be the equation of the boundary of $D$ near $P^{0}$. Assume that $t=0$ is the tangent hyperplane to the boundary of $D$ at $P^{0}$. Therefore $\partial \varphi /\left.\partial x_{i}\right|_{p^{0}}=0$. Let $D$ be on the side $t<\varphi(x)$.

THEOREM 1. If $L u \geqq 0$ in $D$, if $u$ assumes its positive maximum $M$ at $P^{0}$, if

$$
\lim _{P \rightarrow P 0} \frac{\partial u(P)}{\partial x_{i}}=0, \lambda \equiv \lim _{P \rightarrow P 0} \sum a_{i j}(P) \frac{\partial^{2} u(P)}{\partial x_{i} \partial x_{j}} \leqq 0
$$

and if

$$
1+\left.\sum a_{i j} \frac{\partial^{2} \varphi}{\partial x_{i} \partial x_{j}}\right|_{P^{0}}>0
$$

$\varphi \in C^{\prime \prime}$

then $u \equiv M$ in $S\left(P^{0}\right)$.

REMARK 1. Without making any use of (3) one can deduce the following :

Put $\mu \equiv \lim _{P \rightarrow P 0} \sup _{0} \frac{\partial u(P)}{\partial t}(P \in D)$, then $\mu \geqq 0$ since $\mu<0$ will contradict $u\left(P^{0}\right) \geqq u(P)$. Letting $P \rightarrow P^{0}$ in $L u(P) \geqq 0$ and using (2), we obtain $\lambda+$ $a\left(P^{0}\right) M-\mu \geqq 0$, from which it follows that $\lambda \geqq 0$. Since, by (2), $\lambda \leqq 0$, we conclude that $\lambda=0$. Hence $a\left(P^{0}\right) M-\mu \geqq 0$, from which it follows that $\mu \leqq 0$ and, therefore, (since $\mu \geqq 0$ ) $\mu=0$. We also get $a\left(P^{3}\right)=0$.

REMARK 2. The assumptions (2) and (3) can be verified if we assume that $\varphi(x)=o\left(|x|^{2}\right)$ and that $u$ belongs to $C^{\prime \prime}$ in the closure of the domain $V \cap\{t<0\}$, where $V$ is some neighborhood of $P^{0}$. Indeed, by making an appropriate orthogonal transformation we can assume that $a_{i j}\left(P^{0}\right)=\delta_{i j}$. By the mean value theorem we have

$$
u(x, t)-u(0,0)=\sum x_{i} \frac{\partial}{\partial x_{i}} u(\tilde{x}, \tilde{t})+t \frac{\partial}{\partial t} u(\tilde{x}, \tilde{t}) .
$$

Taking $(x, t) \in \bar{D} \cap V \cap\{t<0\}$ such that $|t|=o(|x|)$ and noting that $u(x, t) \leqq$ $u(0,0)$, one can show that $\partial u\left(P^{0}\right) / \partial x_{i}=0$. Noting that $\varphi(x)=o\left(|x|^{2}\right)$ and expanding $[u(x, t)-u(0,0)]$ in terms of the first and second derivatives of $u$, one can show that $\partial^{2} u\left(P^{\jmath}\right) / \partial x_{i}{ }^{2} \leqq 0$, and (2) is thereby proved. The proof of (3) is immediate.

Proof of Theorem 1. For simplicity we shall prove the theorem only in case $n=1$; the proof of the general case is analogous. Lu takes the form

$$
L u \equiv A \frac{\partial^{2} u}{\partial x^{2}}+a \frac{\partial u}{\partial x}+c u-\frac{\partial u}{\partial t} \quad c \leqq 0, A>0 .
$$


From the strong maximum principle [3; Th. 5] it follows that all we need to prove is that $u(P) \equiv M$ if $P \in V^{\prime} \cap S\left(P^{0}\right)$ where $V^{\prime}$ is some neighborhood of $P^{0}$.

There are two possibilities: Either there exists a sequence $\left\{P^{k}\right\}$ such that $P^{k} \in S\left(P^{0}\right), P^{k} \rightarrow P^{0}, u\left(P^{k}\right)=M$, or there exists a neighborhood $V=\left\{x^{2}+t^{2}<R^{2}\right\}$ of $P^{0}$ such that $u(P)<M$ for all $P \in V \cap S\left(P^{0}\right), P \neq P^{0}$. In the first case we can use [3; Th. 5] to conclude that $u(P) \equiv M$ if $P \in V^{\prime} \cap S\left(P^{0}\right)$ where $V^{\prime}$ is some neighborhood of $P^{0}$ (since $u(P)=M$ for all $P \in S\left(P^{k}\right)$ ).

It remains therefore to consider the case in which $u(P)<M$ for all $P \in V \cap S\left(P^{0}\right), P \neq P^{0}$. We shall prove that this case cannot occur by deriving a contradiction. Writing

$$
\varphi(x)=K x^{2}+o\left(x^{2}\right),
$$

we define a domain $D_{\delta}(\delta>0)$ as the intersection of $S\left(P^{0}\right)$ with the set of points $(x, t)$ in $V$ for which

$$
t<\tilde{\varphi}(x)=(K-\delta) x^{2} .
$$

If $K<0$ then, because of (3), we can choose $\delta$ sufficiently small such that

$$
1+\left.A \frac{\partial^{2}}{\partial x^{2}} \tilde{\varphi}(x)\right|_{x=0}>0 .
$$

If $K \geqq 0$, we can obviously take $\delta$ such that $K-\delta<0$ and such that (5) holds.

We now can take $R$ sufficiently small such that $\tilde{\varphi}(x)<\min (0, \varphi(x))$ for all $(x, t)$ in $D_{\delta}, x \neq 0$. Consequently, $u(x, t)<M$ if $t=\tilde{\varphi}(x), x \neq 0$. The function $h(x, t)=-t+\tilde{\varphi}(x)$ vanishes on $t=\tilde{\varphi}(x)$ and is positive in $D_{\delta}$. Therefore, if $\varepsilon>0$ is sufficiently small, then $v=u+\varepsilon h$ is smaller than $M$ at all points on the boundary of $D_{\delta}$ with the exception of $P^{0}$, where $v\left(P^{0}\right)=M$. Noting that $\tilde{\varphi}^{\prime}(0)=0$ and using (5), we conclude that

$$
L h=1+A \tilde{\varphi}^{\prime \prime}(x)+a \tilde{\varphi}^{\prime}(x)+c h>0
$$

if $R$ has been chosen sufficiently small. Hence, $L v=L a+\varepsilon L h>0$. It follows that $v$ cannot assume its positive maximum at interior points of $D_{\delta}$ and, therefore, it assumes its maximum $M$ at $P^{0}$. We thus obtain $\partial v / \partial t \geqq 0$ at $P^{0}$ and, consequently,

$$
\frac{\partial u}{\partial t}=\frac{\partial v}{\partial t}-\varepsilon \frac{\partial h}{\partial t} \geqq \varepsilon>0
$$

(Here

$$
\frac{\partial g}{\partial t}=\liminf _{t \rightarrow 0} \frac{g(0,0)-g(0, t)}{-t} .
$$


On the other hand, letting in (4) $P \rightarrow P^{0}$ in an appropriate way and using (2) and the inequality $L u(P) \geqq 0$, we get

$$
\begin{aligned}
& 0 \leqq \lim A(P) \frac{\partial^{2} u(P)}{\partial x^{2}}+\lim a(P) \frac{\partial u(P)}{\partial x}+C\left(P^{0}\right) M-\lim \sup \frac{\partial u(P)}{\partial t} \leqq \\
& \quad-\lim \sup \frac{\partial u(P)}{\partial t} .
\end{aligned}
$$

We have thus obtained

$$
\limsup _{P \rightarrow P^{0}} \partial u(P) / \partial t \leqq 0<\varepsilon \leqq \partial u / \partial t .
$$

This is however a contradiction (since

$$
\frac{\partial u}{\partial t}=\lim _{t_{k} \rightarrow 0} \frac{\partial u\left(0, t_{k}\right)}{\partial t} \leqq \limsup _{P \rightarrow F^{0}} \frac{\partial u(P)}{\partial t}
$$

for an appropriate sequence $\left\{t_{k}\right\}$ ), and the proof is completed.

REMark (a) Consider the following example: $n=1, P^{0}=(0,0)$ and $D$ defined by

$$
x^{2}+t^{2}<R, t<\gamma_{1} x, t<\gamma_{2} x \quad \gamma_{1}>0>\gamma_{2} .
$$

The function $u(x, t)=\left(t-\gamma_{1} x\right)\left(\gamma_{2} x-t\right)$ satisfies the following properties : $u<0$ in $D, u=0$ at $P^{0}$, and

$$
L u \equiv A \frac{\partial^{2} u}{\partial x^{2}}+a \frac{\partial u}{\partial x}-\frac{\partial u}{\partial t}=-2 A \gamma_{1} \gamma_{2}+0(|x|+|t|) \geqq 0,
$$

provided $R$ is sufficiently small. Consequently, (3) and the second assumption in (2) are not satisfied and also the assertion of Theorem 1 is false.

REMARK (b). Consider now the case in which the tangent hyperplane at $P^{0}$ is not of the form $t=$ const.. We shall prove that in this case Theorem 1 is false. Take $n=1$ and consider first the case in which $D$ is defined by

$$
x>0, x^{2}+t^{2}<R^{2} .
$$

If $L u \equiv \partial^{2} u / \partial x^{2}-\partial u / \partial t$, then the function $u(x, t)=-x$ takes its maximum in $\bar{D}$ at $P^{0}=(0,0), L u=0$, but $u \neq 0$ in $S\left(P^{0}\right)$.

Consider next the case in which $\bar{D}$ is defined by

$$
x>\alpha t, x^{2}+t^{2}<R^{2} .
$$

and take $L u=\partial^{2} u / \partial x^{2}-\alpha \partial u / \partial x-\partial u / \partial t$. 
The transformation $t^{\prime}=t, x^{\prime}=x-\alpha t$ carries the present case into the previous one.

Note that if the tangent hyperplane $H$ at $P^{0}$ is not the plane $t=0$ and the axes are rotated so as to give $H$ the equation $t^{\prime}=0$ (in new $x^{\prime}$, $t^{\prime}$ coordinate), then $L u$ loses the form (1), for $u_{x^{\prime} t^{\prime}}$ and $u_{t^{\prime} t^{\prime}}$ will appear ir it.

REMARK (c). If in Theorem 1 the domain $D$ is on the side $t>\varphi(x)$, then the theorem is false. Indeed, as a counter-example take $u=-t$, and $D$ bounded from below by $t=0$.

\section{Consider the linear operator}

$$
\begin{array}{rlrl}
L^{\prime} u \equiv & \sum_{i, j=1}^{n} a_{i j}(x, t) \frac{\partial^{2} u}{\partial x_{i} \partial x_{j}}+\sum_{i, j=1}^{m} b_{i j}(x, t) \frac{\partial^{2} u}{\partial t_{i} \partial t_{j}}+\sum_{i=1}^{n} a_{i}(x, t) & \frac{\partial u}{\partial x_{i}} \\
& +\sum_{i=1}^{m} b_{i}(x, t) \frac{\partial u}{\partial t_{i}}+a(x, t) u & & a(x, t) \leqq 0,
\end{array}
$$

where $x=\left(x_{1}, \cdots, x_{n}\right)$ and $t=\left(t_{1}, \cdots, t_{m}\right)$ vary in the closure of a given $(n+m)$-dimensional domain $D$. We assume that $L^{\prime}$ is elliptic in the variables $x$ and parabolic in the variables $t$, that is, for every real vector $\lambda \neq 0$,

$$
\sum a_{i j} \lambda_{i} \lambda_{j}>0, \quad \sum b_{i j} \lambda_{i} \lambda_{j} \geqq 0
$$

All the coefficients appearing in (6) are assumed to be continuous in $\bar{D}$ and $u$ is assumed to be continuous in $\bar{D}$ and to have a continuous $t$ derivative and continuous second $x$-derivatives in $D$. Under these assumptions, Nirenberg [3; Th. 2] has proved a weak maximum principle from which it follows that, if $L^{\prime} u \geqq 0$ in $D$ then $u$ must assume its positive maximum on the boundary.

Let $P^{0}=\left(x^{0}, t^{0}\right)$ be a point on the boundary of $D$ such that $u\left(P^{0}\right)=$ $M>0$ is the maximum of $u$ in $\bar{D}$. Assume that there exists a neighborhood $V:\left|x-x^{0}\right|^{2}+\left|t-t^{0}\right|^{2}<R_{0}^{2}$ of $P^{0}$ such that $u(x, t)<M$ in $V \cap D$. We then can prove the following theorem.

THEOREM 2. If there exists a sphere $S:\left|x-x^{\prime}\right|^{2}+\left|t-t^{\prime}\right|^{2}<R^{2}$ passing through $P^{0}$ and contained in $\bar{D}$, and if $x^{0} \neq x^{\prime}$ then, under the assumptions made above (in particular, $L^{\prime} u \geqq 0, u(x, t)<M$ in $V \cap D$ ), every nontangential derivative $\partial u / \partial \tau$ at $\left(x^{0}, t^{0}\right)$, understood as the limit inferior of $\Delta u, \Delta \tau$ along a non-tangential direction $\tau$, is negative.

By a non-tangential direction we mean a direction from $P^{0}$ into the interior of the sphere $S$. 
REMARK (a). If $a(x, t) \equiv 0$ then the assumption $M>0$ is superflous.

REMARK (b). In $\S 3$ we shall show that the assumption $x^{0} \neq x^{\prime}$ is essential. We shall also discuss the case in which $u(x, t)$ is not smaller than $M$ at all the points of $V \cap D$.

Proof. For simplicity we give the proof in the case $m=n=1$, so that

$$
L^{\prime} u \equiv A \frac{\partial^{2} u}{\partial x^{2}}+B \frac{\partial^{2} u}{\partial t^{2}}+a \frac{\partial u}{\partial x}+b \frac{\partial u}{\partial t}+c u \quad A>0, B \geqq 0, c \leqq 0
$$

the proof of the general case is quite similar. Without loss of generality we can take $\left(x^{\prime}, t^{\prime}\right)=(0,0)$ and $x^{0}>0$. Furthermore, we may assume that, with the exception of $P^{0}, S$ lies in $V \cap D$, so that $u(x, t)<M$ in $S-P^{0}$. Denote by $C$ the intersection of $S$ with the plane $x>\delta$, where $0<\delta<x^{0}$. The function

$$
h(x, t)=\exp \left(-\alpha\left(x^{2}+t^{2}\right)\right)-\exp \left(-\alpha R^{2}\right)
$$

satisfies the following properties : $h=0$ on the boundary of $S, h \geqq 0$ in $C$; if $\alpha$ is large enough, then

$$
\begin{aligned}
L^{\prime} h= & \exp \left(-\alpha\left(x^{2}+t^{2}\right)\right)\left[4 \alpha^{2}\left(A x^{2}+B t^{2}\right)-2 \alpha(A+B+a x+b t)+c\right] \\
& -c \exp \left(-\alpha R^{2}\right)>0 .
\end{aligned}
$$

(Here we used $x>\delta>0, c \leqq 0$.)

If $\varepsilon$ is sufficiently small, then the function $v=u+\varepsilon h$ is smaller than $M$ at all points of the boundary of $C$ with the exception of $P^{0}$, where $v\left(P^{0}\right)=M$. Since $L^{\prime} v=L^{\prime} u+\varepsilon L^{\prime} h>0, v$ cannot assume its positive maximum in $\bar{C}$ at the interior of $C$ (since, otherwise, at such interior points $L^{\prime} v$ would be non-positive). Hence, $v$ assumes its maximum at $P^{0}$ and, consequently, $\partial v / \partial \tau=\lim \inf (\Delta v / \Delta \tau) \leqq 0$. Since along the normal $\nu$ (i. e., along the radius through $\left.P^{0}\right) \partial h / \partial \nu>0$ and since along the tangential direction $\sigma \partial h / \partial \sigma=0$, it follows that $\partial h / \partial \tau>0$. Using the definition of $v$, we conclude that $\partial u / \partial \tau=\partial v / \partial \tau-\varepsilon \partial h / \partial \tau<0$, and the proof is completed.

Added in proof. Theorem 2 was recently and independently proved also by R. Viborni, On properties of solutions of some boundary value problems for equations of parabolic type, Doklody Akad. Nauk SSSR, 117 (1957), 563-565.

3. From now on we shall consider only parabolic operators of the form (1). Suppose the assumption $u<M$ in $V \cap D$, made in Theorem 2, is replaced by $u \leqq M$. If there exists a sequence of points $\left\{P^{k}\right\}$ such 
that $P^{k} \rightarrow P^{0}, P^{k} \in D, P^{k}=\left(x^{k}, t^{k}\right)$ and $t^{k} \geqq t^{0}, u\left(P^{k}\right)=M$, then, by [3; Th. 5], $u \equiv M$ in $S\left(P^{k}\right)$. Hence, if the boundary of $D$ near $P^{0}$ is sufficiently smooth, $u \equiv M$ in some set $V^{\prime} \cap D$ where $V^{\prime}$ is some neighborhood of $P^{0}$. Consequently $\partial u / \partial \tau=0$ for every $\tau$.

If $u(P) \leqq M$ for all $P \in V \cap D$, if $u(P)$ is not strictly smaller than $M$ for all $P \in V \cap D, P \neq P^{0}$, and if the previous situation does not arise, then one and only one of the following cases must occur:

(i) $u<M$ at all points $(x, t)$ in $V \cap D$ with $t \geqq t^{0}$. Using [3; Th. 5] one can easily conclude that there exists a neighborhood $V^{\prime}$ of $P$ such that $u<M$ in $V^{\prime} \cap D$, and Theorem 2 remains true.

(ii) $u<M$ at all points $(x, t)$ in $V \cap D$ with $t>t_{0}$ and $u \equiv M$ at all points $(x, t)$ in $V \cap D$ with $t \geqq t_{0}$. We then consider only those directions $\tau$ along which $u<M$. We claim that Theorem 2 is not true for the present situation. To prove this, consider the following simple counterexample :

$$
P^{0}=(0,0), M=0, L u=\frac{\partial^{2} u}{\partial x^{2}}-\frac{\partial u}{\partial t}, u(x, t)=\left\{\begin{array}{cll}
-t^{2} & \text { if } t>0 \\
0 & \text { if } t<0 .
\end{array} .\right.
$$

$u$ satisfies $L u \geqq 0$ and assumes its maximum 0 for $t \leqq 0$. But, the derivative $\partial u / \partial \tau$ at $P^{0}=(0,0)$, along any direction $\tau$, is zero.

As another counter-example (with $L u=0$ ) one can take a fundamental solution of the heat equation.

Note that the preceding counter-examples are valid without any assumptions on the behavior of the boundary of $D$ near $P^{0}$.

We shall now consider the case $\boldsymbol{x}^{1}=\boldsymbol{x}^{0}$ which was excluded by the assumptions of Theorem 2. We shall assume that at $P^{0}=(0,0)$ there passes a tangent hyperplane $t=0$. If $D$ is above this hyperplane, then the preceding counter-examples show that Theorem 2 is not true. It remains to consider the case in which $D$ is " essentially" below $t=0$, that is, if we denote by $t=\varphi(x)$ the equation of the boundary of $D$ near $P^{0}$, then $D$ is on the side $t<\varphi(x)$. In this case, however, Theorem 1 tells us that in general we cannot assume both $u\left(P^{0}\right)=\max u(P)>0$ $(P \in \bar{D})$ and $u<u\left(P^{0}\right)$ in $V \cap D$.

The example in $\S 1$ Remark (a) can also serve as a counter-example to Theorem 2 in case $P^{0}$ is a vertex-point. Indeed, along the $t$-direction

$$
\left.\frac{\partial u}{\partial t}\right|_{P^{0}}=\left.\frac{\partial}{\partial t}\left[\left(t-\gamma_{1} x\right)\left(\gamma_{2} x-t\right)\right]\right|_{x=0, t=0}=0 \text {. }
$$

By a small modification of this counter-example one can get a counter-example to the analogue of Theorem 2 for elliptic operators [2] [4] in case $P^{0}$ is a vertex. Indeed, define $D$ by

$$
x^{2}+y^{2}<R^{2}, y<\gamma_{1} x, y>\gamma_{2} x
$$


and take $L u=\partial^{2} u / \partial x^{2}+A \partial^{2} u / \partial y^{2}$, where $A>\left|\gamma_{1} \gamma_{2}\right|$. The function $u(x, y)=$ $\left(y-\gamma_{1} x\right)\left(y-\gamma_{2} x\right)$ satisfies : $u<0$ in $D, u=0$ at the origin, $L u=2 \gamma_{1} \gamma_{2}+2 A>0$. But along any direction $\tau$ within $D, \partial u /\left.\partial \tau\right|_{x=0, y=0}=0$.

4. Let $D$ be a domain bounded by the two hyperplanes $t=0, t=$ $T>0$ and a surface $B$ between them. Assume that the intersection $\{t=T\} \cap \bar{D}$ is the closure of an open set on $t=T$, and denote by $A$ the boundary of $D$ on $t=0$. The Neumann problem for the parabolic equation $L u=0$ consists in finding a solution to the equation $L u=0$ which satisfies the following initial and boundary conditions:

$$
u=f \text { on } A, \frac{\partial u}{\partial \nu}=g \text { on } B
$$

( $f, g$ are given functions).

From Theorem 2 and from the strong maximum principle [3; Th. 5] we conclude: If. for every point $P^{0}=\left(x^{0}, t^{0}\right)$ of $B$ (i) there exists a sphere with center $\left(x^{\prime}, t^{\prime}\right), \quad x^{\prime} \neq x^{0}$, passing through $P^{0}$ and contained in $\bar{D}$, and (ii) $\overline{S\left(P^{0}\right)}$ contains interior points of $A$, then the Neumann problem has at most one solution. Clearly, this uniqueness property holds also for the more general problem where $\partial u / \partial \nu$ is replaced by $\partial u / \partial \tau$ and $\tau$ is a nontangential direction which varies on $B$.

As another application to Theorem 2, one can deduce the positivity of $\partial G / \partial \nu$, where $G$ is the Green's function of $L u=0$.

5. Let $\boldsymbol{D}$ be a domain bounded by $t=0, t=T(0<T \leqq \infty)$ and surfaces $\Gamma_{k}, 0 \leqq k \leqq m, \Gamma_{0}$ being the outer boundary. Suppose further that the intersection of each $\Gamma_{k}$ with $t=t_{0}\left(0 \leqq t_{0}<T\right)$ is a simple closed curve $\gamma_{k}\left(t_{j}\right)$ which belongs to $C^{(3)}$ and does not reduce to a single point. Write $u_{x_{i}}=\partial u / \partial x_{i}, u_{t}=\partial u / \partial t$. We shall consider the following problem $P$ :

$$
\sum_{i, j=1}^{n} a_{i j}(x, t) u_{x_{i} x_{j}}-u_{t}=c(x, t, u, \nabla u)
$$

(where $\nabla u$ denotes the vector $\partial u / \partial x_{i}$ ),

$$
\begin{array}{ccc}
\frac{\partial u}{\partial \tau} \equiv \sum_{i=1}^{n} \alpha_{i}(x, t) u_{x_{i}}+\alpha(x, t) u_{t}=\varphi(x, t, u) & (x, t) \in \Gamma=\sum_{k=0}^{m} \Gamma_{k} \\
u(x, 0)=\psi(x) \text { on } A & A=\bar{D} \cap\{t=0\}
\end{array}
$$

We make the following assumptions:

(a) $a_{i j}(x, t)$ is continuous in $\bar{D} ; c(x, t, u, \nabla u)$ and it first derivatives with respect to $u, \nabla u$ are continuous for $(x, t) \in \bar{D}$ and for all values of $u, \nabla u$. 
(b) $\varphi$ and $\partial \varphi / \partial u$ are continuous for all $(x, t) \in \Gamma$ and for all $u$.

( c ) $\alpha_{i}(x, t), \alpha(x, t)$ are continuous for $(x, t) \in \Gamma ; \psi(x)$ is continuous in $A$.

(d) (9) is parabolic in $\bar{D}$, that is, there exists a positive constant $\delta$ such that

$$
\sum a_{i j}(x, t) \xi_{i} \xi_{j} \geqq \delta \sum \xi_{i}^{2}
$$

holds for all real $\xi$ and for all $(x, t) \in \bar{D}$.

(e ) On each surface $\Gamma_{k}(k=0,1, \cdots, m)$ either all the directions $\tau=\left(\alpha_{i}\right.$,

$\alpha$ ) are exterior or all are interior, and in the exterior case $\alpha \geqq 0$ and the directions $\left(\alpha_{i}, 0\right)$ are exterior while in the interior case $\alpha \leqq 0$ and the directions $\left(\alpha_{i}, 0\right)$ are interior.

Denote by $\sum$ the class of functions $u(x, t)$ defined and continuous in $\bar{D}$ and satisfying the following conditions:

( $\alpha) \quad \partial u / \partial t, \partial u / \partial x_{i}, \partial^{2} u / \partial x_{i} \partial x_{j}$ are continuous in $D$;

( $\beta$ ) For every $R>0, \partial u / \partial x_{i}$ is bounded in $D \cap\left\{|x|^{2}+t^{2}<R^{2}\right\}$.

THEOREM 3. Under the assumptions (a)-(e) the problem $P$ cannot have two different solutions in the class $\Sigma$.

We shall need the following lemma.

Lemma. There exists a function $\zeta(x)$ defined in $A$ and having the following properties: (i) $\zeta$ has continuous first derivatives in $A$ and continuous second derivatives in the interior of $A$; (ii) $\partial \zeta / \partial \nu=-1$ and $\partial \zeta / \partial \mu=0$ on $\gamma_{0}(0), \cdots, \gamma_{m}(0)$, where $\partial / \partial \nu$ and $\partial / \partial \mu$ denote the derivatives with respect to the interior normal and to any tangential direction, respectively.

Proof of the Lemma. It will be enough to construct a function $\chi_{0}(x)$ which is $C^{\prime \prime}$ in $A$, which vanishes in a neighborhood of $\gamma_{i}(0)(i=1$, $\cdots, m)$ and for which $\partial \chi_{0} / \partial_{\nu}=-1, \partial \chi_{0} / \partial \mu=0$ along $\gamma_{0}(0)$; constructing $\gamma_{1}(x)$ in a similar manher, we can then take $\zeta(x)=\sum \chi_{1}(x)$. Since $\gamma_{0}(0)$ belongs to $C^{(3)}$, the normals issuing from $\gamma_{0}(0)$ and inwardly directed cover in a one-to-one manner a small inner neighborhood of $\gamma_{0}(0)$, call it $A_{0}$. To each point $x$ in $A_{0}$ there corresponds a unique point $x^{\prime \prime}$ on the boundary of $\gamma_{0}(0)$, such that $x$ lies on the normal through $x^{3}$. Denote by $\sigma(x)$ the distance $\left|x-x^{0}\right|$. It is elementary to show that $\sigma(x)$ has continuous second derivatives in $A_{0}$. Denote by $A_{1}$ the domain $0 \leqq \sigma \leqq \varepsilon_{0}$, where $\varepsilon_{0}>0$ is small enough so that $A_{1} \subset A_{0}$. The function

$$
\chi_{0}(x)=\left\{\begin{array}{cc}
\frac{1}{3 \varepsilon_{0}{ }^{2}}\left(\varepsilon_{0}-\sigma(x)\right)^{3} & \text { if } x \in \bar{A}_{1} \\
0 & \text { if } x \in A-A_{1}
\end{array}\right.
$$

belongs to $C^{\prime \prime}$ in $A$ and satisfies : $\partial \chi_{0} / \partial \nu=\partial \chi_{0} / \partial \sigma=-1$ and $\partial \chi_{0} / \partial \nu=0$ on $\gamma_{0}(0)$, and $\chi_{0}$ vanishes near $\gamma_{k}(0),(1 \leqq k \leqq m)$; the proof is completed. 
Proof of Theorem 3. We first consider the case $n>1$. We may suppose that the vectors $\left(\alpha_{i}, \alpha\right)$ are exterior directions on $\Gamma_{0}, \cdots, \Gamma_{q}$ and that $\left(\alpha_{i}, \alpha\right)$ are interior directions on $\Gamma_{q+1}, \cdots, \Gamma_{m}$. Suppose now that $u$ and $v$ are two solutions in $\Sigma$ of the problem $P$, and define $w=v-u$. Writing

$$
\begin{aligned}
C(x, t, u, v) & =\int_{0}^{1} \frac{\partial}{\partial u} c(x, t, u+\lambda w, \nabla u+\lambda \nabla w) d \lambda \\
C_{i}(x, t, u, v) & =\int_{0}^{1} \frac{\partial}{\partial u_{x_{i}}} c(x, t, u+\lambda w, \nabla u+\lambda \nabla w) d \lambda \\
\Phi(x, t, u, v) & =\int_{0}^{1} \frac{\partial}{\partial u} \varphi(x, t, u+\lambda w) d \lambda
\end{aligned}
$$

and using (9), (10) and (11), we obtain for $w$ the following system :

$$
\begin{gathered}
\sum a_{i j} w_{x_{i} x_{j}}-w_{t}=C w+\sum C_{i} w_{x_{i}} \\
\frac{\partial w}{\partial \tau}=\sum \alpha_{i} w_{x_{i}}+\alpha w_{t}=\Phi w \\
w(x, 0)=0 .
\end{gathered}
$$

Substituting $w(x, t)=z(x, t) \exp (K t+M \zeta(x))$, where $\zeta(x)$ is the function constructed in the lemma and $K, M$ are constant to be determined later, we get for $z$ the following system :

$$
\begin{gathered}
\sum a_{i j} z_{x_{i} x_{j}}-z_{t}=-M \sum a_{i j} \zeta_{x_{i} x_{j}} z-M^{2} \sum a_{i j} \zeta_{x_{i}} \zeta_{x_{j}} z \\
-2 M \sum a_{i j} \zeta_{x_{i}} z_{x_{j}}+K z+C z+M \sum C_{i} \zeta_{x_{i}} z+\sum C_{i} z_{x_{i}} \\
\frac{\partial z}{\partial \tau} \equiv \sum \alpha_{i} z_{x_{i}}+\alpha z_{t}=-M \sum \alpha_{i} \zeta_{x_{i}} z-\alpha K z+\Phi z \\
z(x, 0)=0 .
\end{gathered}
$$

If $0 \leqq k \leqq q, \alpha \geqq 0$ and $\sum \alpha_{i}(x, 0) \zeta_{x_{i}}(x)>0$ on $\gamma_{k}(0)$, since the angle between the vectors $\left(\alpha_{i}\right)$ and grad $\zeta$ is $<\pi / 2$. By continuity we get $\sum \alpha_{i}(x, t) \zeta_{x_{i}}(x) \geqq$ $\eta>0$ on $\gamma_{k}(t)$, provided $0 \leqq t \leqq T^{\prime}$ and $T^{\prime}$ is sufficiently small. Hence, we can choose $M$ sufficiently large such that

$$
-M \sum \alpha_{i} \zeta_{x_{i}}-\alpha K+\Phi<0
$$

holds on $\gamma_{k}(t)$, provided $K \geqq 0$ and $0 \leqq t \leqq T^{\prime}$.

If $q+1 \leqq k \leqq m, \alpha \leqq 0$ and $\sum \alpha_{i}(x, 0) \zeta_{x_{i}}(x)<0$, since the angle between $\left(\alpha_{i}\right)$ and $-\operatorname{grad} \zeta$ is $<\pi / 2$. Again, if $K \geqq 0$ and $M$ is sufficiently large, then

$$
-M \sum \alpha_{i} \zeta_{x_{i}}-\alpha K+\Phi>0
$$


on $\gamma_{k}(t), 0 \leqq t \leqq T^{\prime}$.

Having fixed $M$, we now choose $K$ sufficiently large so that the coefficient of $z$ on the right side of $\left(13^{\prime}\right)$ becomes positive in the domain $D_{T^{\prime}}=D \cup\left\{0<t<T^{\prime}\right\}$. We claim that $z \equiv 0$ in $D_{T^{\prime}}$. Indeed, if this is not the case then, using $\left(15^{\prime}\right)$ and the weak maximum principle [ $3 ; \mathrm{Th} .2$ ] we conclude that $z$ assumes either its positive maximum or its negative minimum on the boundary $\sum_{k=0}^{m} \gamma_{k}(t), 0 \leqq t \leqq T^{\prime}$, of $D_{T^{\prime}}$. It will be enough to consider the case in which $z$ assumes its positive maximum at a point $P^{0}$ on $\gamma_{k}(t)$. If $0 \leqq k \leqq q$, then $\partial z / \partial \tau \geqq 0$ since $\tau$ is outwardly directed. On the other hand, using (14') and (16) we get $\partial z / \partial \tau<0$, which is a contradiction. If $q+1 \leqq k \leqq m$, then $\partial z / \partial \tau \leqq 0$ since $\tau$ is inwardly directed. On the other hand, using $\left(14^{\prime}\right)$ and (17) we get $\partial z / \partial \tau>0$ which is a contradiction. We have thus proved that $z \equiv w \equiv 0$ in $D_{T^{\prime}}$. We can now apply a classical procedure of continuation and thus complete the proof of the theorem for the case $n>1$.

In the case $n=1, \Gamma=\Gamma_{0}$ is composed of two curves $\Gamma_{01}$ aud $\Gamma_{02}$. Suppose $\Gamma_{0 k}$ intersects $t=0$ at $a_{k}, a_{1}<a_{2}$. The function

$$
\zeta(x)=\frac{\left(x-a_{1}\right)\left(x-a_{2}\right)}{a_{2}-a_{1}}
$$

can be used in the preceding proof. Note that it is not necessary to make any assumptions on the smoothness of the curves $\Gamma_{0 k}$.

\section{REFERENCES}

1. F. A. Ficken, Uniqueness theorems for certain parabolic equations, J. Rational Mechanics and Analysis, 1 (1952), 573-578.

2. E. Hopf, A remark on linear elliptic differential equations of second order, Proc. Amer. Math. Soc., 3 (1952), 791-793.

3. L. Nirenberg, A strong maximum principle for parabolic equations, Comm. Pure and Appl. Math., 6 (1953), 167-177.

4. O. A. Olainik, On properties of some boundary problems for equations of elliptic type, Mat. Sb. (N.S.), 30 (72) (1952), 695-702.

INDIANA UNIVERSITY 



\section{PACIFIC JOURNAL OF MATHEMATICS}

\section{EDITORS}

\section{H. L. Royden}

Stanford University

Stanford, California

\section{R. A. Beaumont}

University of Washington

Seattle 5 , Washington

\author{
A. L. Whiteman
}

University of Southern California

Los Angeles 7, California

E. G. Straus

University of California

Los Angeles 24, California

\section{ASSOCIATE EDITORS}
E. F. BECKENBACH
A. HORN
L. NACHBIN
G. SZEKERES
C. E. BURGESS
V. GANAPATHY IYER
I. NIVEN
F. WOLF
M. HALL
R. D. JAMES
T. G. OSTROM
E. HEWITT
M. S. KNEBELMAN
M. M. SCHIFFER
K. YOSIDA

\section{SUPPORTING INSTITUTIONS}

\author{
UNIVERSITY OF BRITISH COLUMBIA \\ CALIFORNIA INSTITUTE OF TECHNOLOGY \\ UNIVERSITY OF CALIFORNIA \\ MONTANA STATE UNIVERSITY \\ UNIVERSITY OF NEVADA \\ OREGON STATE COLLEGE \\ UNIVERSITY OF OREGON \\ UNIVERSITY OF SOUTHERN CALIFORNIA
}

\author{
STANFORD UNIVERSITY \\ UNIVERSITY OF UTAH \\ WASHINGTON STATE COLLEGE \\ UNIVERSITY OF WASHINGTON \\ * * * * \\ AMERICAN MATHEMATICAL SOCIETY \\ CALIFORNIA RESEARCH CORPORATION \\ HUGHES AIRCRAFT COMPANY \\ THE RAMO-WOOLDRIDGE CORPORATION
}

Mathematical papers intended for publication in the Pacific Journal of Mathematics should be typewritten (double spaced), and the author should keep a complete copy. Manuscripts may be sent to any of the editors. All other communications to the editors should be addressed to the managing editor, E. G. Straus at the University of California, Los Angeles 24, California.

50 reprints per author of each article are furnished free of charge; additional copies may be obtained at cost in multiples of 50 .

The Pacific Journal of Mathematics is published quarterly, in March, June, September, and December. The price per volume (4 numbers) is $\$ 12.00$; single issues, $\$ 3.50$. Back numbers are available. Special price to individual faculty members of supporting institutions and to individual members of the American Mathematical Society: $\$ 4.00$ per volume; single issues, $\$ 1.25$.

Subscriptions, orders for back numbers, and changes of address should be sent to Pacific Journal of Mathematics, 2120 Oxford Street, Berkeley 4, California.

Printed at Kokusai Bunken Insatsusha (International Academic Printing Co., I.td.), No. 10, 1-chome, Fujimi-cho, Chiyoda-ku, Tokyo, Japan.

PUBLISHED BY PACIFIC JOURNAL OF MATHEMATICS, A NON-PROFIT CORPORATION

The Supporting Institutions listed above contribute to the cost of publication of this Journal, but they are not owners or publishers and have no responsibility for its content or policies. 


\section{Pacific Journal of Mathematics}

\section{Vol. 8, No. 2 \\ April, 1958}

John Herbert Barrett, Second order complex differential equations with a real independent variable ............................ 187

Avner Friedman, Remarks on the maximum principle for parabolic equations and its applications ......................... 201

Richard Robinson Goldberg, An inversion of the Stieltjes transform ....... 213

Olavi Hellman, On the periodicity of the solution of a certain nonlinear integral equation .................................. 219

Gilbert Helmberg, A theorem on equidistribution on compact groups...... 227

Lloyd Kenneth Jackson, Subfunctions and the Dirichlet problem ......... 243

Naoki Kimura, The structure of idempotent semigroups. I ............ 257

Stephen Kulik, A method of approximating the complex roots of equations........................................ 277

Ancel Clyde Mewborn, A note on a paper of L. Guttman.............. 283

Zeev Nehari, On the principal frequency of a membrane ............ 285

G. Pólya and I. J. Schoenberg, Remarks on de la Vallée Poussin means and convex conformal maps of the circle ...................... 295

B. M. Stewart, Asymmetry of a plane convex set with respect to its centroid .......................................... 335

Hans F. Weinberger, Lower bounds for higher eigenvalues by finite difference methods

Edwin Weiss and Neal Zierler, Locally compact division rings ......... 369

Bertram Yood, Homomorphisms on normed algebras ................. 373 